\title{
«Fehlerdatenbank»
}

Seitens der Foederatio medicorum scrutantium FMS wurde in den letzten Wochen der Vorwurf erhoben, die TARMED-Tarifstruktur enthalte mehr als 2000 Leistungen, die den Patienten gefährden bzw. eine qualitativ hochstehende Medizin verhindern würden. Diese Verlautbarungen haben ihren Weg auch in die Medien und politischen Kreise gefunden.

Der Verhandlungsdelegation wie auch dem ZV sind diese > 2000 "gefährlichen Leistungen» nicht bekannt. Einzig an der Ärztekammer vom 13. Dezember 2001 wurden vereinzelte Beispiele genannt, die aber nicht erhellend waren, weil sie nicht Zeugnis von Gefährlichkeit, vielmehr aber von den Schwierigkeiten der Sprechenden ablegten, die Tarifstruktur richtig zu verstehen.

Zentralvorstand und Verhandlungsdelegation nehmen diese Vorwürfe sehr ernst und wollen ihnen auf den Grund gehen. Wir eröffnen deshalb eine FMH-Mitgliedern in CUG-Form zugängliche Datei, in der von jedem Mitglied unter Namensangabe und mit Begründung solche angeblich patienten- bzw. qualitätsgefährdende Leistungen eingebracht werden können.

Die Datenbank ist direkt elektronisch zugänglich (www.tarmed.ch $\rightarrow$ Forum $\rightarrow$ CUG); Sie können uns Ihre Anmerkungen aber auch schriftlich (FMH Tarifdienst, Burghöhe 1, 6208 Oberkirch) mitteilen.

Wir danken Ihnen für Ihre Mitarbeit.

Dr. med. H. H. Brunner, Präsident FMH

\section{«Une banque des données erronées»}

Durant ces dernières semaines, la Foederatio medicorum scrutantium (FMS) nous a adressé le reproche suivant lequel la structure tarifaire TARMED contenait plus de 2000 prestations susceptibles de mettre en danger le patient et d'empêcher l'exercice d'une médecine qualitative de pointe. Ces informations ont également été retransmises par les médias et les milieux politiques.

La délégation aux négociations TARMED de la FMH et le Comité central n'ont pas connaissance de ces 2000 et quelque prestations. Lors de la Chambre médicale du 13 décembre 2001, quelques exemples ont bien été mentionnés. Ne prouvant aucune mise en danger, ces derniers n'ont toutefois pas apporté les éclaircissements désirés. Ils témoignaient plutôt de la difficulté des orateurs de bien comprendre la structure tarifaire.

Le Comité central et la délégation aux négociations prennent toutefois ces reproches très au sérieux. Ils souhaitent les examiner à fond. C'est la raison pour laquelle nous créons, sous forme d'un "Closed User Group» (CUG), une banque de données accessible à tous les membres de la FMH. Pour autant qu'il décline son identité et justifie sa prise de position, chacun pourra y entrer les positions du tarif qu'il considère comme mettant en danger patient et qualité des prestations. Cette banque de données est accessible directement par voie électronique sous le site www.tarmed.ch $\rightarrow$ Forum $\rightarrow$ CUG. Vous pouvez aussi nous faire parvenir vos remarques par écrit (à envoyer au Service tarifaire de la FMH, Burghöhe 1, 6208 Oberkirch).

Nous vous remercions de votre précieuse collaboration.

Dr H. H. Brunner, président de la FMH 